\title{
Genomic characterization of Haemophilus influenzae: a focus on the capsule locus
}

\author{
Caelin C. Potts ${ }^{1}$, Nadav Topaz ${ }^{2}$, Lorraine D. Rodriguez-Rivera ${ }^{3}$, Fang Huํ․ How-Yi Chang ${ }^{3}$, Melissa J. Whaley ${ }^{1}$, \\ Susanna Schmink ${ }^{1}$, Adam C. Retchless ${ }^{1}$, Alexander Chen ${ }^{1}$, Edward Ramos ${ }^{4}$, Gregory H. Doho ${ }^{4}$ and Xin Wang ${ }^{1 *}$
}

\begin{abstract}
Background: Haemophilus influenzae $(\mathrm{Hi})$ can cause invasive diseases such as meningitis, pneumonia, or sepsis. Typeable Hi includes six serotypes (a through f), each expressing a unique capsular polysaccharide. The capsule, encoded by the genes within the capsule locus, is a major virulence factor of typeable Hi. Non-typeable (NTHi) does not express capsule and is associated with invasive and non-invasive diseases.
\end{abstract}

Methods: A total of 395 typeable and 293 NTHi isolates were characterized by whole genome sequencing (WGS). Phylogenetic analysis and multilocus sequence typing were used to characterize the overall genetic diversity. Pairwise comparisons were used to evaluate the capsule loci. A WGS serotyping method was developed to predict the Hi serotype. WGS serotyping results were compared to slide agglutination (SAST) or real-time PCR (rt-PCR) serotyping.

Results: Isolates of each Hi serotype clustered into one or two subclades, with each subclade being associated with a distinct sequence type (ST). NTHi isolates were genetically diverse, with seven subclades and 125 STs being detected. Regions I and III of the capsule locus were conserved among the six serotypes ( $\geq 82 \%$ nucleotide identity). In contrast, genes in Region II were less conserved, with only six gene pairs from all serotypes showing $\geq 56 \%$ nucleotide identity. The WGS serotyping method was $99.9 \%$ concordant with SAST and 100\% concordant with rt-PCR in determining the Hi serotype.

Conclusions: Genomic analysis revealed a higher degree of genetic diversity among NTHi compared to typeable Hi. The WGS serotyping method accurately predicted the Hi capsule type and can serve as an alternative method for $\mathrm{Hi}$ serotyping.

Keywords: Haemophilus influenzae, Whole genome sequencing, Capsule locus, Serotype, Genetic diversity, Multilocus sequence typing

\section{Background}

Haemophilus influenzae (Hi) can cause severe and lifethreatening invasive diseases, especially in persons $<5$ or $\geq 65$ years old [1-3]. There are six different Hi serotypes (Hia, Hib, Hic, Hid, Hie and Hif), which each express a unique polysaccharide capsule, as well as nontypeable $\mathrm{Hi}$ (NTHi) strains, which lack capsule expression. Since the implementation of the Hib vaccine, the burden of Hib disease has decreased dramatically in the United States [1]. However, NTHi and other non-b serotypes

\footnotetext{
*Correspondence: gqe8@cdc.gov

'Bacterial Meningitis Laboratory, Meningitis and Vaccine Preventable Diseases Branch, Division of Bacterial Diseases, National Center for Immunization and Respiratory Diseases, Centers for Disease Control and Prevention, 1600 Clifton Rd NE, Mailstop H17-2, Atlanta, GA 30329, USA

Full list of author information is available at the end of the article
}

continue to cause disease. For example, invasive Hia disease has exhibited more than a two-fold increase in incidence between the time periods 2002-2008 and 2009-2015 [3], highlighting the continued need to monitor the distribution of Hi serotypes.

Hi was one of the first organisms with a complete genome sequenced and its genomic characterization has provided important insights into our fundamental understanding of bacterial genomics [4-8]. The genetic diversity for $\mathrm{Hi}$ has predominantly been characterized through multilocus sequence typing (MLST) [9]. MLST demonstrated that each Hi serotype was associated with a few sequence types (STs), while NTHi isolates were associated with multiple STs [9-11]. Two main groups were identified: Group I contained isolates from NTHi

(c) The Author(s). 2019 Open Access This article is distributed under the terms of the Creative Commons Attribution 4.0 International License (http://creativecommons.org/licenses/by/4.0/), which permits unrestricted use, distribution, and 
and serotypes Hia, Hib, Hic, and Hid, while Group II contained Hia, Hie and Hif isolates [9-11]. More recently, WGS studies confirmed that NTHi isolates were more diverse, with multiple phylogenetic clades detected [12-14].

The Hi polysaccharide capsule is encoded by the capsule locus, which is composed of three regions [15]. Regions I and III are conserved among all six serotypes, while the Region II genes are unique to each serotype [15]. Region I contains the bex $A B C D$ operon and Region III includes the $h c s A$ and $h c s B$ genes, which are involved in exporting capsule polysaccharides [16-18]. Region II contains three to eight genes, depending on the serotype, and is required for polysaccharide synthesis [19-24].

Slide agglutination serotyping (SAST) and real-time PCR (rt-PCR) serotyping are commonly used to identify Hi serotypes. SAST detects polysaccharide expression using serotype-specific antibodies, while rt-PCR detects the presence of a single, serotype-specific gene [25-27]. Serotype-determination by SAST can be affected by variations in technique and the lot of antisera used [28]. Multiple reports have indicated that rt-PCR is a preferred method for serotyping because it is highly sensitive and can confirm both typeable and NTHi isolates [29, 30].

In this report, the genetic diversity of $688 \mathrm{Hi}$ isolates was assessed using whole genome sequencing (WGS). In addition, a comprehensive analysis of each capsule gene was conducted to develop a WGS serotyping method to predict Hi capsule expression. Finally, the concordance between the WGS serotyping method and each of the two conventional serotyping methods, SAST or rt-PCR, was assessed in determining Hi serotypes.

\section{Results}

\section{H. influenzae genetic diversity}

The genetic relatedness of $688 \mathrm{Hi}$ isolates was determined using a maximum likelihood phylogeny. The isolates overall clustered by serotype and formed three large clades (I, II, and III, Fig. 1). Clade I was divided into three subclades, containing predominantly Hia, Hie, or Hif isolates. Clade II contained seven subclades that contain Hia, Hib, Hic, Hid, or NTHi isolates. In contrast, clade III contained only NTHi isolates, which clustered into five separate subclades. Consistent with MLST analysis, clades II and III (Group I by MLST) were more closely related to each other than to clade I (Group II by MLST) $[9,10,31]$.

A higher degree of genetic diversity was observed among NTHi than the typeable isolates. Isolates of each serotype were predominantly associated with a single subclade, with the exception of Hia and Hib. Isolates of Hia and Hib each formed two subclades. The two Hib subclades were both detected within clade II and the two Hia subclades were in clades I and II. The Hia isolates in clade II were more tightly clustered with Hib

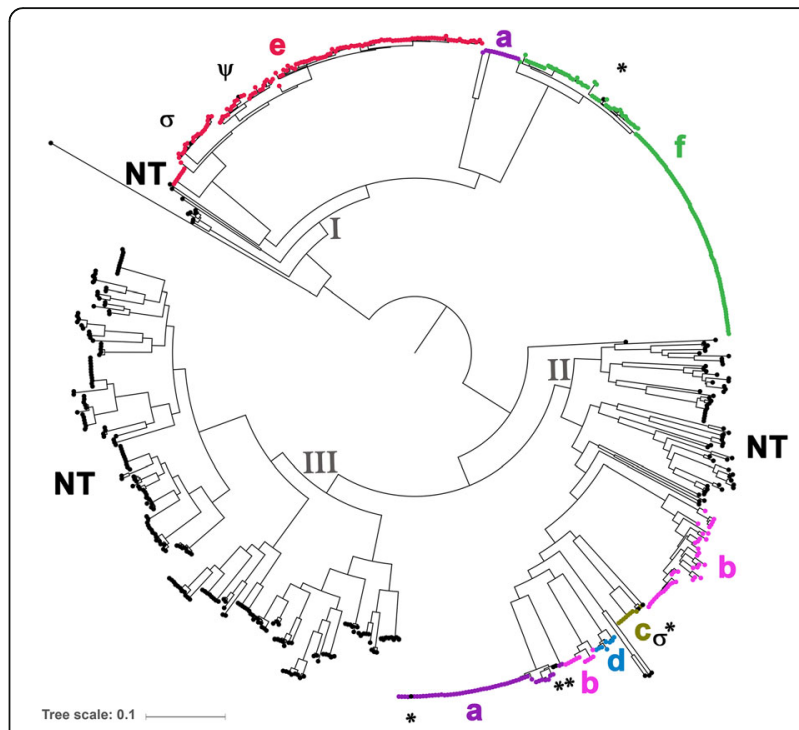

Fig. 1 Population structure of $H$. influenzae isolates. The genomic relatedness of $688 \mathrm{Hi}$ isolates is depicted as a maximum likelihood phylogeny. The three main clades (I, II, and III) are labeled on the tree and each isolate is color coded by the serotype, as determined by slide agglutination. The NTHi isolates within the serotype-specific subclades are denoted by $\psi, \sigma$, or * The $\psi$ indicates the isolate that had discrepant serotyping results between WGS and SAST methods. The $\sigma$ indicates the only two NTHi isolates that contained an internal stop codon within a capsule gene. The ${ }^{*}$ indicates the remaining five NTHi isolates (capsule nulls) that were detected within serotypespecific subclades. The tree scale is 0.1 substitutions per site along the length specified. Bootstrap values were 100/100 for all major nodes

and Hid than Hic isolates, while the clade I Hia isolates were more tightly clustered with Hif than the Hie isolates. In contrast, the NTHi isolates clustered into seven separate subclades within clades II and III. The majority of NTHi isolates clustered together in clade II and III, with only eight NTHi isolates being clustered with typeable isolates within the serotype-specific subclades as denoted by $*, \psi$, and $\sigma$ in Fig. 1.

To understand the genetic differences between the two Hia or Hib subclades, pair-wise gene comparisons were conducted to identify unique genes that distinguish between the two Hia or Hib subclades. For Hia, two genes were present in the clade I Hia isolates but absent from the isolates in clade II. An additional 57 unique genes were present in the clade II Hia isolates that were absent in the clade I isolates (Additional file 2: Table S1). For Hib, 47 genes were present in the large Hib subclade isolates but absent from all the isolates in the small Hib subclade. (Additional file 2: Table S2).

Consistent with the phylogenetic analysis, greater genetic diversity was detected among NTHi isolates by MLST, with more sequence types (STs) among NTHi compared to typeable Hi (Table 1 and Additional file 2: Table S3). Of the 284 NTHi isolates with a ST assigned, 
Table 1 MLST distribution within each serotype

\begin{tabular}{|c|c|}
\hline Sequence Type & Number of Isolates \\
\hline \multicolumn{2}{|l|}{ Hia - clade I } \\
\hline ST-62 & 13 \\
\hline Other & 1 \\
\hline \multicolumn{2}{|l|}{ Hia - clade II } \\
\hline ST-23 & 7 \\
\hline ST-56 & 30 \\
\hline ST-576 & 10 \\
\hline Other & 6 \\
\hline \multicolumn{2}{|c|}{ Hib - small clade (most similar to Hid) } \\
\hline ST-222 & 6 \\
\hline Other & 5 \\
\hline \multicolumn{2}{|l|}{ Hib - large clade } \\
\hline ST-6 & 21 \\
\hline Other & 17 \\
\hline \multicolumn{2}{|l|}{$\mathrm{Hic}$} \\
\hline ST-9 & 7 \\
\hline Other & 2 \\
\hline \multicolumn{2}{|l|}{ Hid } \\
\hline ST-10 & 5 \\
\hline Other & 3 \\
\hline \multicolumn{2}{|l|}{ Hie } \\
\hline ST-18 & 44 \\
\hline ST-66 & 22 \\
\hline ST-121 & 8 \\
\hline ST-127 & 8 \\
\hline ST-386 & 5 \\
\hline ND (no fuck gene) & 33 \\
\hline Other & 12 \\
\hline \multicolumn{2}{|l|}{ Hif } \\
\hline ST-124 & 122 \\
\hline Other & 9 \\
\hline \multicolumn{2}{|l|}{ NTHi } \\
\hline ST-3 & 13 \\
\hline ST-11 & 6 \\
\hline ST-12 & 7 \\
\hline ST-14 & 11 \\
\hline ST-34 & 6 \\
\hline ST-57 & 8 \\
\hline ST-103 & 11 \\
\hline ST-107 & 9 \\
\hline ST-139 & 8 \\
\hline ST-143 & 5 \\
\hline ST-145 & 6 \\
\hline ST-155 & 5 \\
\hline
\end{tabular}

Table 1 MLST distribution within each serotype (Continued)

\begin{tabular}{ll}
\hline Sequence Type & Number of Isolates \\
\hline ST-156 & 8 \\
ST-165 & 5 \\
ST-182 & 5 \\
ND (no fuck gene) & 7 \\
Other & 172 \\
\hline
\end{tabular}

$40 \%$ were identified as one of the 15 STs that were detected in $\geq 5$ NTHi isolates (Table 1). In contrast, each serotype was predominantly associated with one or a few STs that differed by only one allele (Table 1 ). For example, the Hic, Hid, and Hif isolates were associated with ST-9, ST-10, and ST-124, respectively; the Hie isolates were associated with five genetically related STs (ST-18, ST-66, ST-121, ST-127, and ST-386). Notably, the two distinct subclades of Hia or Hib were each associated with STs that differed at all seven MLST loci. The clade I Hia isolates were ST-62 and the clade II Hia isolates were predominantly one of three genetically related STs (ST-23, ST-56 or ST-576). For Hib, the small subclade was ST-222 and the larger subclade was ST-6. Furthermore, 6/8 NTHi isolates that were detected within typeable subclades had the predominant ST associated with that subclade. For example, the two NTHi isolates in the Hie subclade were ST-66 and ST-18. A ST could not be determined for 33 Hie and 7 NTHi isolates because each isolate was missing the fucK gene required for the MLST analysis.

Sequence types (STs) are shown for each serotype. STs detected in fewer than five isolates are grouped as "Other" and denoted in Additional file 2: Table S3. Isolates missing the fucK gene could not be assigned a ST and are denoted by ND (not determined).

\section{Genetic diversity of the $\boldsymbol{H}$. influenzae capsule locus}

A reference database containing the alleles for each capsule gene was generated using a genome from each serotype (a total of six genomes). The reference database contained 64 alleles: six alleles for each of the six genes in Regions I and III (36 alleles total) and a single allele for each of the 28 Region II genes (Fig. 2). This reference database was queried against all $688 \mathrm{Hi}$ genomes, resulting in the identification of 275 unique alleles in the three regions of the capsule locus (Fig. 3).

The allelic diversity was assessed for each capsule gene using pair-wise comparisons for all alleles detected among the 688 genomes (Fig. 3). The capsule genes in Regions I and III exhibited similar levels of sequence diversity with $>82.7 \%$ identity, except bexD, which was more conserved with $>90.3 \%$ identity (Fig. 3a). A gene-based, neighbor-net phylogenetic analysis demonstrated that the sequence 


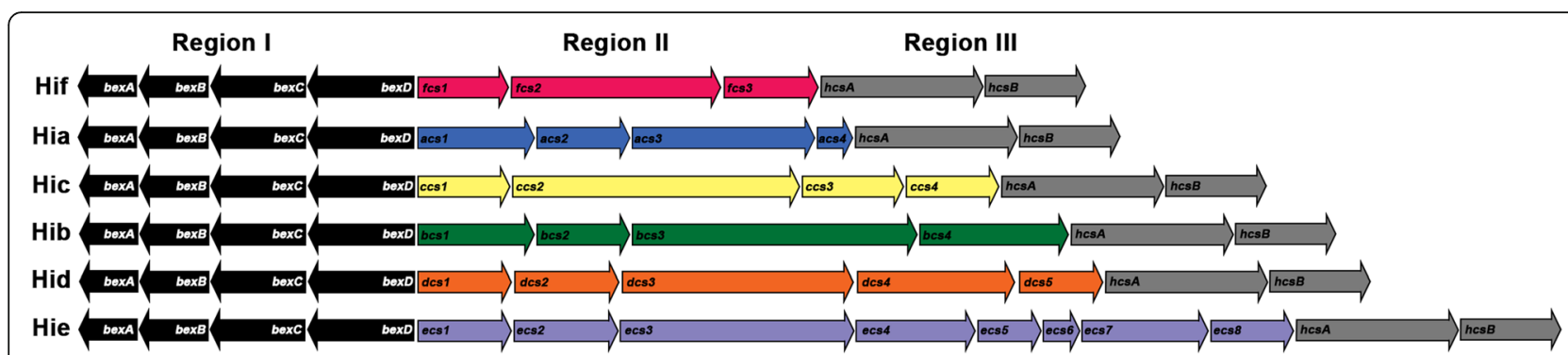

Fig. 2 Genetic organization of the H. influenzae capsule genes. All serotypes contained the Region I genes bexABCD (black) and the Region III genes (gray): hcsA and hcsB. The Region II genes were divergent among serotypes. Each arrow represents a different gene and is labeled with the gene name. The Region II arrows are color coded by serotype

diversity within Regions I and III was sufficient to differentiate each of the six serotypes (Additional file 1: Figure S1). The serotype-specific Region II genes exhibited high sequence similarity within each serotype, with only a few alleles detected per gene (Fig. 3b). The Region II genes of Hia and Hib exhibited a lower degree of sequence similarity compared to all other serotypes. However, if only alleles detected within the same Hia or Hib subclade were considered, the sequence similarity was more comparable to other serotypes (Additional file 1: Figure S2).

Finally, the inter-serotype sequence diversity of the Region II genes was determined using pair-wise

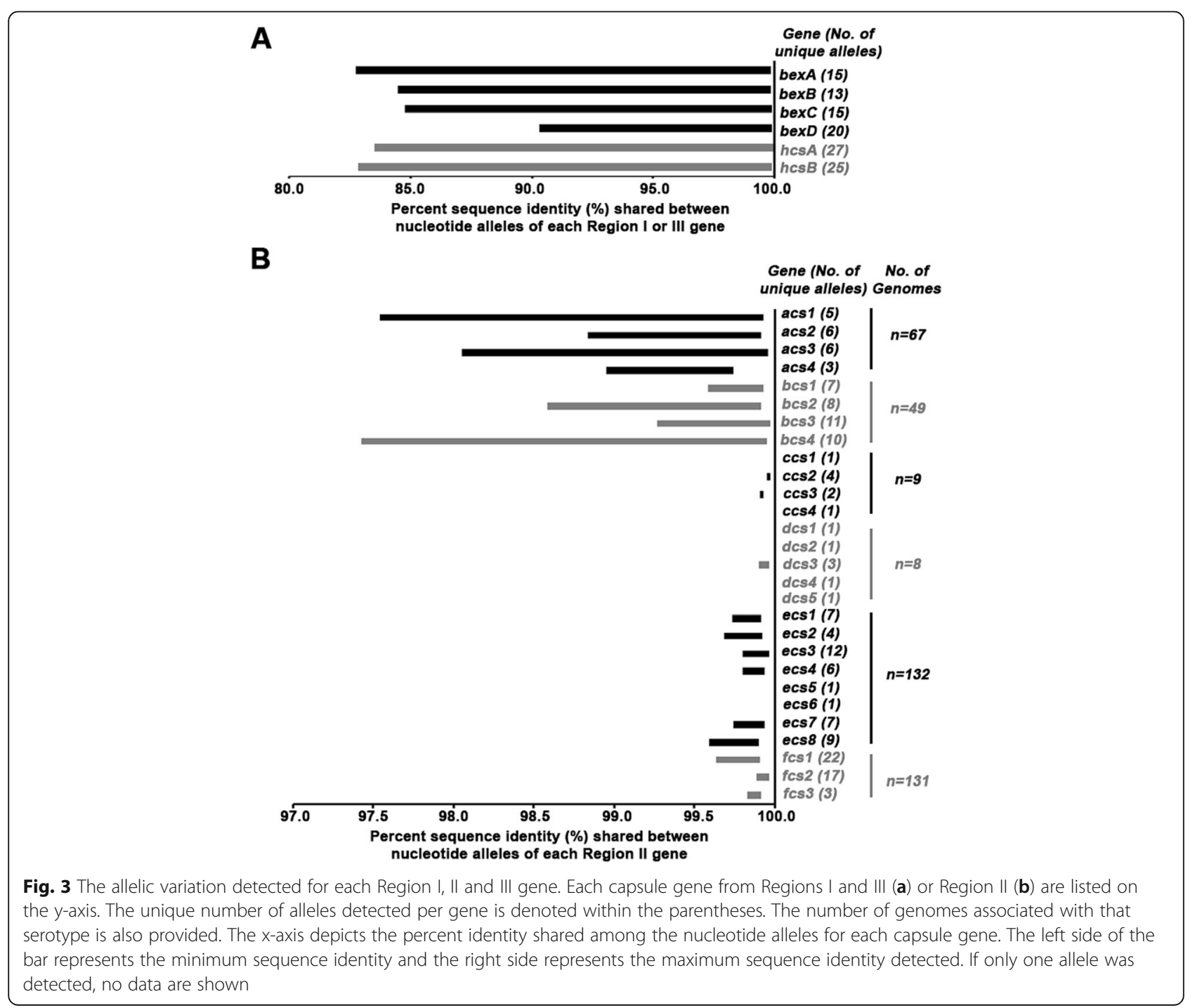


comparisons for every possible gene pair. Only six gene pairs exhibited significant homology (Table 2). Three gene pairs (acs2/bcs2, ccs1/fcs1 and dcs5/ecs 8$)$ had moderate sequence identity $(<88 \%)$ and three gene pairs (acs1/bcs1, dcs1/ecs1, and dcs2/ecs2) had high sequence identity (>92\%). The sequence identity between acs1 and $b c s 1$ was comparable to the amount of allelic variation observed for acs 1 alone.

WGS serotyping is an effective method for predicting the $H$. influenzae capsule type

An in silico method was developed to predict the $\mathrm{Hi}$ serotype using the following three steps (Fig. 4). First, the capsule genes within the assembly were identified using the 64 allele reference database as the query. Then, the sequence intactness of each capsule gene was assessed to predict the likelihood of expression, and finally, the prediction tool assigned a serotype. Using this method, the serotype was predicted for each of the 688 isolates, resulting in the identification of 396 typeable and 292 NTHi. Nearly all NTHi isolates (290/292) had a capsule null locus, completely lacking Region I, II and III genes. The remaining two NTHi isolates contained an internal stop codon in either ccs 2 or ecs 3 (denoted by $\sigma$ in Fig. 1).

The concordance between the WGS serotyping method and SAST or rt-PCR was assessed. High concordance (99.9\%) was observed between WGS and SAST in serotyping 688 isolates. One hundred percent concordance was observed between WGS and rt-PCR in serotyping 496 isolates (Table 3). Only one isolate was identified as NTHi by SAST but Hie by both WGS and rt-PCR (denoted by $\psi$ in Fig. 1 ).

\section{Discussion}

In this report, 688 invasive $\mathrm{Hi}$ isolates, representing all six capsule types, were characterized by WGS. The genomic analysis presented in this study was consistent with previous studies $[9,10,12,31]$. Overall, a higher degree of genetic diversity was observed among NTHi than the typeable isolates. All 688 isolates clustered into three large clades, corresponding to Groups I and II, which

Table 2 Inter-serotype Sequence Identity between Region II Gene Pairs

\begin{tabular}{lcc}
\hline Gene Names & Minimum Similarity & Maximum Similarity \\
\hline acs1/bcs1 & 96.13 & 98.39 \\
acs2/bcs2 & 56.81 & 62.33 \\
ccs1/fcs1 & 85.11 & 85.39 \\
dcs1/ecs1 & 92.62 & 92.89 \\
dcs2/ecs2 & 94.08 & 94.23 \\
dcs5/ecs8 & 86.81 & 87.01 \\
\hline
\end{tabular}

were previously defined by MLST [9, 10, 31]. However, MLST may not be optimal for typing Hi because a proportion of isolates (25\% of Hie and $2 \%$ of NTHi isolates) did not have the fucK gene required for MLST analysis. This also has important implications for rt-PCR, because fucK was also identified as a species-specific target for the detection of $\mathrm{Hi}$ [32].

Subclades associated with a specific serotype or NTHi were also identified. While no typeable isolates were observed within the NTHi-specific subclades, eight NTHi isolates were detected within serotype-specific subclades. Notably, six of the eight NTHi isolates within the serotype-specific subclades had the ST most commonly detected within the typeable isolates. It is possible that these six NTHi isolates represent strains that recently lost their capsule expression. However, capsule loss may be a rare event; otherwise one would expect to observe more NTHi in serotype specific clades.

The high resolution WGS methods used in this study provided novel insights into the genomic distinctions among the two Hia and Hib subclades. Each subclade was associated with a distinct ST and a number of unique genes. The presence of unique genes, which were further confirmed using a representative, intact genome generated by PacBio sequencing, may explain the difference between the two Hia or Hib subclades. An association between the distinct Hia and Hib subclades and specific alleles for the Region I and III genes was also observed. This finding could indicate that an ancestral strain specifically acquired the Region II genes from a Hia or Hib strain and expanded to form these subclades. However, the directionality of this potential capsule acquisition remains unclear. While the biological relevance of the two Hia or Hib subclades is still unclear, the two subclades of the same serotype had no direct association with syndromes or age.

Characterization of the capsule genes from WGS data enabled the development of an in silico method for accurately predicting the serotype, which was highly concordant with SAST and rt-PCR. Two other WGS methods for determining the Hi serotype have been recently described [14, 33]. However, this is the first comprehensive characterization of the capsule locus using a large isolate collection that includes a comparative analysis of all three serotyping methods. Consistent with the previous findings, Regions I and III are relatively conserved among all six serotypes and Region II genes are mostly unique to each $\mathrm{Hi}$ serotype. Interestingly, some of the Region II genes shared homology among serotypes. The sequences of $a c s 1$ and bcs 1 were highly similar and both genes have CDP-ribitol pyrophosphorylase activity in vitro, which could indicate they are functionally redundant $[19,20]$. Two other gene pairs (dcs1/ecs 1 , and $d c s 2 / e c s 2)$ also exhibited high sequence identity but 


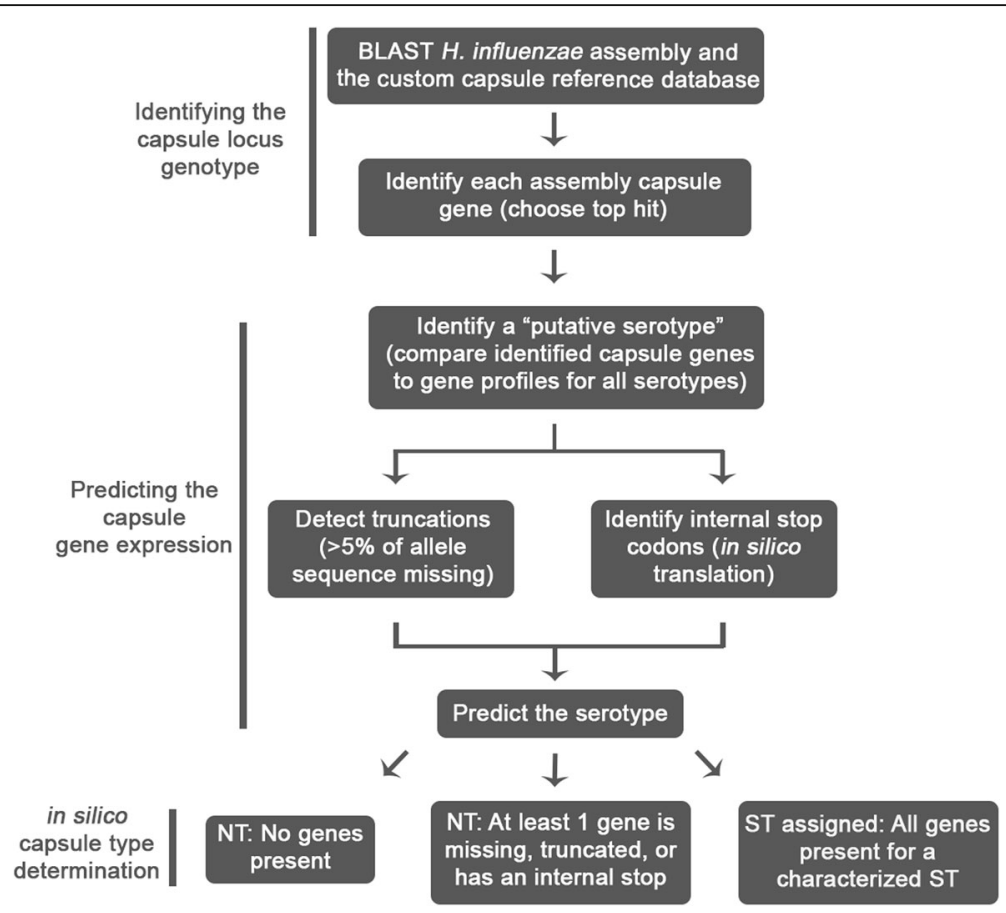

Fig. 4 WGS serotyping method for determining H. influenzae serotype from WGS data. The three main steps of this process include identifying the capsule genes, predicting the capsule gene expression, and assigning the predicted serotype

because only a few Hid genomes were available for the study, the amount of allelic variation within each Hid gene remains unclear. Additional complementation studies are required to determine if these ORF pairs within Region II could be classified as the same gene.

High concordance was observed for the three different methods used to determine the Hi serotype. Only one discrepant isolate was detected, which was NTHi by SAST but classified as Hie by WGS and rt-PCR. There could be multiple possible explanations for this observation. This isolate could express the Hie capsule as predicted by WGS, but at an undetectable level by SAST.
Alternatively, this isolate could be truly non-typeable because the expression of the polysaccharide has been disrupted by a genetic element outside the coding regions assessed by either the rt-PCR or WGS methods. Additional studies are required to determine the cause of this discrepancy. Because of the high concordance observed among the three assays used in this study, the volume of $\mathrm{Hi}$ isolates, the cost attributed to each assay, and the potential need for additional genomic characterization must be considered when selecting the appropriate method for serotype identification.

Table 3 Concordance of the WGS serotyping method and other serotyping methods

\begin{tabular}{|c|c|c|c|c|c|c|}
\hline \multirow[t]{2}{*}{ Serotype* } & \multicolumn{3}{|c|}{ Concordance: SAST and WGS } & \multicolumn{3}{|c|}{ Concordance: rt-PCR and WGS } \\
\hline & Total Isolates & No. of Concordant Isolates & Percent & Total Isolates & No. of Concordant Isolates & Percent \\
\hline a & 67 & 67 & 100.0 & 64 & 64 & 100.0 \\
\hline b & 49 & 49 & 100.0 & 46 & 46 & 100.0 \\
\hline c & 9 & 9 & 100.0 & 9 & 9 & 100.0 \\
\hline$d$ & 8 & 8 & 100.0 & 8 & 8 & 100.0 \\
\hline e & 131 & 131 & 100.0 & 122 & 122 & 100.0 \\
\hline$f$ & 131 & 131 & 100.0 & 131 & 131 & 100.0 \\
\hline NT & 293 & 292 & 99.7 & 116 & 116 & 100.0 \\
\hline Total & 688 & 687 & 99.9 & 496 & 496 & 100.0 \\
\hline
\end{tabular}

Serotype* defined by SAST method. The concordance data is reported as the number and percent of isolates. Only one discordant isolate was identified 


\section{Conclusions}

This study provided a large-scale genomic characterization of invasive $\mathrm{Hi}$ isolates representing all capsule types. Whole genome phylogenetic analysis demonstrated that the isolates clustered into three large clades, with typeable isolates forming distinct, serotype-specific subclades. The in-depth characterization of the capsule locus highlighted that Regions I and III are highly conserved among serotypes, while very little homology was observed between Region II genes. Finally, a novel WGS method was developed to determine the Hi serotype, demonstrating that the capsule gene content is a strong and accurate predictor of capsule expression.

\section{Methods}

\section{H. influenzae culture and serotyping}

$\mathrm{Hi}$ isolates were collected from 1990 to 2017 through surveillance programs. A total of 675 isolates were collected from 27 U.S. states and 13 isolates were obtained from three different countries. All serotypes were represented: Hia $n=67$, Hib $n=49$, Hic $n=9$, Hid $n=8$, Hie $n=131$, Hif $n=131$, NTHi $n=293$. Hi isolates were cultured on chocolate II agar with hemoglobin and IsoVitaleX (BD BBL) at $37{ }^{\circ} \mathrm{C}$ with $5 \% \mathrm{CO}_{2}$. The serotype was determined by SAST using serotype-specific antisera obtained from Remel and by rt-PCR as described previously $[28,34,35]$.

\section{Whole genome sequencing and phylogenetic analysis}

DNA was extracted manually using the Gentra Puregene yeast/bacteria DNA extraction kit (Qiagen) or with a chemagic Prepito instrument (PerkinElmer) using the Cyto Pure Kit. Genomic libraries were generated using the NEBNext Ultra DNA kit according to manufacturer instructions. Sequencing was completed on a MiSeq or HiSeq 2500 using $250 \mathrm{bp}$ paired-end reads. Raw reads were filtered and trimmed using cutadapt, version 1.8.1 [36], and assembled into genomes using SPAdes, version 3.7.0 [37]. A subset of genomes were generated using PacBio methods as described previously [38]. The dataset supporting the conclusions of this article is available in the NCBI repository, [Bioproject: PRJNA512636].

The maximum likelihood phylogenetic tree was generated using whole genome data and kSNP3 [39]. The tree was annotated as graphics using the iTol program [40]. All genes within each assembly were identified by comparing the sequences against the PubMLST H. influenzae collection [41]. The neighbor-net phylogenetic network for the Region I and III capsule genes was generated using SplitsTree4 after aligning the genes with MUSCLE [42, 43]. BLAST was used to identify the MLST loci present within each assembly; allele numbers and STs were assigned with PubMLST $[41,44]$. To identify the genes that distinguished the two subclades for Hia or Hib, gene by gene comparisons were conducted and gene function was assigned using InterProScan 5 [45]. The presence or absence of genes among isolates from each subclade were compared using a custom in-house python script. In addition, all reported, differentially detected genes were manually curated to confirm the absence of any BLAST results within a representative PacBio assembly from that subclade.

\section{Characterization of the $\boldsymbol{H}$. influenzae capsule locus genes}

A reference database containing at least one allele for each Hi capsule gene was generated. Reciprocal best hits (BLAST) was used to identify the capsule locus alleles within a GenBank genome sequence for each serotype: Hia (CP077811), Hib (FQ312006), Hic (HQ651151) [21], Hid (HQ424464) [21], Hie (FM882247) [22], and Hif (CP005967). Additional refinement was completed by manually curating the start and stop sites of each open reading frame. All of the capsule alleles identified through this process were compiled into a custom reference database. BLAST was used to identify the capsule alleles within the 688 isolate genomes using the custom reference database as a query.

To assess the homology among genes within the capsule locus, pair-wise comparisons were completed using BLAST and the percent sequence identity was reported [44]. If genes varied in length, the longer allele was used as the query sequence. To determine the allelic sequence diversity, all identified alleles for each Region I, II or III gene were compared. For inter-serotype comparisons, every possible Region II gene pair, across all serotypes, was assessed.

\section{Prediction of $\boldsymbol{H}$. influenzae serotype from WGS data}

Each genome assembly was compared against the custom reference database using BLAST [44]. All BLAST hits with $90 \%$ or greater identity to an allele in the reference database were pooled. If two genes matched the same region of the genome, the gene with the lower alignment score (defined as (Identity*Alignment Length)/Allele Length) was discarded to ensure preference was given to exact matches rather than longer, inexact matches. To detect potential internal stop codons, in silico translations were completed using the BioPython Seq module [46].

To predict capsule type, the top hits for each assembly were compared to the known essential genes for each serotype. The capsule genotype was assigned based on the highest number of corresponding genes identified. To predict the capsule expression, factors that could impact expression were considered: missing genes, premature internal stops, and truncated genes (those that had $95 \%$ or less coverage when aligned against its closest reference). If all genes were present and intact, the serotype 
was predicted to be the serotype with the highest number of identified genes. Non-typeable (NTHi) was assigned to assemblies containing internal stops, truncated or missing capsule genes. The capsule prediction method related to this project has been made publicly available (https:// github.com/ntopaz/hinfluenzae_capsule_characterization).

\section{Assessing concordance among three serotyping assays} Concordance between SAST and WGS was calculated using all 688 isolates; concordance between rt-PCR and WGS was assessed using the 496 isolates (Hia $n=64$, Hib $n=46$, Hic $n=9$, Hid $n=8$, Hie $n=123$, Hif $n=131$, NTHi $n=115$ as defined by SAST methods) that had been tested by rt-PCR. Any isolate identified to have a discrepant result was re-tested by the discrepant method for confirmation. Only confirmed discrepant isolates were included.

\section{Supplementary information}

Supplementary information accompanies this paper at https://doi.org/10. 1186/s12864-019-6145-8

Additional file 1: Figure S1. Neighbor-net phylogenetic analysis of the Region I and III alleles. A gene-based neighbor-net phylogenetic analysis was generated by aligning the alleles of the Region I and III genes. The sequence diversity present within the Region I and III genes was sufficient to differentiate the isolates from each of the six serotypes, including the distinct Hia and Hib subclades. Figure S2. The allelic variation detected within and between subclades for Hia and Hib Region II genes. The $\mathrm{x}$-axis depicts the percent identity shared among alleles for each capsule gene. The left side of the bar represents the minimum sequence identity and the right side represents the maximum sequence identity detected. Allelic variation was quantified for either alleles detected within the same subclade (intra-subclade) or between the two subclades (inter-subclade).

Additional file 2: Tables S1 and S2. Genes differentially detected between the two Hia subclades (Table S1) or the two Hib subclades (Table S2). A value of 1 indicates $100 \%$ of the isolates in that subclade contained the gene; a value of 0 indicates that the gene was detected in $0 \%$ of isolates within that subclade. Table $\mathbf{S 3}$. Sequence types detected in $<5$ isolates. $\mathrm{ND}=$ Not determined.

\section{Abbreviations}

Hi: Haemophilus influenzae; Hia: Haemophilus influenzae serotype a; Hib: Haemophilus influenzae serotype b; Hic: Haemophilus influenzae serotype c; Hid: Haemophilus influenzae serotype d; Hie: Haemophilus influenzae serotype e; Hif: Haemophilus influenzae serotype f; MLST: Multilocus sequence typing; NTHi: Non-typeable Haemophilus influenzae; rt-PCR: Real-time polymerase chain reaction; SAST: Slide agglutination serotyping;

ST: Sequence type; WGS: Whole genome sequencing

\section{Acknowledgements}

We would like to thank the U.S. state health departments for providing the strains used in this study. We would like to thank the Office of Advanced Molecular Detection and the Biotechnology Core Facility Genome Sequencing Laboratory for their help and support. We thank the Laboratory Leadership Service Fellowship for their support of C.C. Potts during this study. And finally, we would like to thank our colleagues in the Bacterial Meningitis Laboratory, including our summer student Chandler Annesi, and the MVPDB epidemiology team for their support.

\section{Disclaimer}

The findings and conclusions in this report are those of the authors and do not necessarily represent the official position of the Centers for Disease Control and Prevention.

\section{Authors' contributions}

Data acquisition and analysis was completed by CCP, NT, LDR-R, FH, H-YC, MJW, SS, ACR, AC, and ER. Interpretation of data was completed by CCP, NT, $A C R, G H D$, and XW. Design of the work was completed by CCP, NT and XW. CCP drafted the work and all co-authors provided substantive review. All authors have read and approved the manuscript.

\section{Funding}

This work was supported by the Centers for Disease Control and Prevention. The funding agency supported the reagents and personnel to complete the study but had no role in the study design, data collection and interpretation, or the decision to submit the work for publication.

\section{Availability of data and materials}

The dataset generated during the current study is available in the NCBI repository, [Bioproject: PRJNA512636]. The capsule prediction method related to this project has been made publicly available (https://github.com/ntopaz/ hinfluenzae_capsule_characterization).

\section{Ethics approval and consent to participate}

Institutional Review Board review was not required by any participating institutions. The strains were collected for disease surveillance and CDC determined that this study was non-research.

\section{Consent for publication}

Not applicable.

\section{Competing interests}

The authors declare that they have no competing interests.

\section{Author details}

'Bacterial Meningitis Laboratory, Meningitis and Vaccine Preventable Diseases Branch, Division of Bacterial Diseases, National Center for Immunization and Respiratory Diseases, Centers for Disease Control and Prevention, 1600 Clifton Rd NE, Mailstop H17-2, Atlanta, GA 30329, USA. ${ }^{2}$ CDC Foundation, Atlanta, GA, USA. ${ }^{3}$ IHRC Inc., Atlanta, GA, USA. ${ }^{4}$ CSRA, Inc., Atlanta, GA, USA.

Received: 15 March 2019 Accepted: 26 September 2019

Published online: 12 October 2019

\section{References}

1. MacNeil JR, Cohn AC, Farley M, Mair R, Baumbach J, Bennett N, et al. Current epidemiology and trends in invasive Haemophilus influenzae disease--United States, 1989-2008. Clin Infect Dis. 2011;53(12):1230-6.

2. Blain A, MacNeil J, Wang X, Bennett N, Farley MM, Harrison LH, et al. Invasive Haemophilus influenzae Disease in Adults $>/=65$ Years, United States, 2011. Open Forum Infect Dis. 2014:1 (2):ofu044.

3. Soeters HM, Blain A, Pondo T, Doman B, Farley MM, Harrison LH, et al. Current Epidemiology and Trends in Invasive Haemophilus influenzae Disease-United States, 2009-2015. Clin Infect Dis. 2018;67(6):881-9.

4. Fleischmann RD, Adams MD, White O, Clayton RA, Kirkness EF, Kerlavage AR, et al. Whole-genome random sequencing and assembly of Haemophilus influenzae Rd. Science. 1995;269(5223):496-512.

5. Tatusov RL, Mushegian AR, Bork P, Brown NP, Hayes WS, Borodovsky M, et al. Metabolism and evolution of Haemophilus influenzae deduced from a whole-genome comparison with Escherichia coli. Curr Biol. 1996;6(3):279-91.

6. Harrison A, Dyer DW, Gillaspy A, Ray WC, Mungur R, Carson MB, et al. Genomic sequence of an otitis media isolate of nontypeable Haemophilus influenzae: comparative study with $\mathrm{H}$. influenzae serotype d, strain KW20. J Bacteriol. 2005;187(13):4627-36.

7. Hogg JS, Hu FZ, Janto B, Boissy R, Hayes J, Keefe R, et al. Characterization and modeling of the Haemophilus influenzae core and supragenomes based on the complete genomic sequences of Rd and 12 clinical nontypeable strains. Genome Biol. 2007;8(6):R103.

8. Boissy R, Ahmed A, Janto B, Earl J, Hall BG, Hogg JS, et al. Comparative supragenomic analyses among the pathogens Staphylococcus aureus, Streptococcus pneumoniae, and Haemophilus influenzae using a modification of the finite supragenome model. BMC Genomics. 2011;12:187.

9. Meats E, Feil EJ, Stringer S, Cody AJ, Goldstein R, Kroll JS, et al. Characterization of encapsulated and noncapsulated Haemophilus 
influenzae and determination of phylogenetic relationships by multilocus sequence typing. J Clin Microbiol. 2003;41(4):1623-36.

10. Connor TR, Corander J, Hanage WP. Population subdivision and the detection of recombination in non-typable Haemophilus influenzae. Microbiology. 2012;158(Pt 12):2958-64.

11. LaCross NC, Marrs CF, Gilsdorf JR. Population structure in nontypeable Haemophilus influenzae. Infect Genet Evol. 2013;14:125-36.

12. De Chiara M, Hood D, Muzzi A, Pickard DJ, Perkins T, Pizza M, et al. Genome sequencing of disease and carriage isolates of nontypeable Haemophilus influenzae identifies discrete population structure. Proc Natl Acad Sci U S A. 2014;111(14):5439-44.

13. Staples M, Graham RMA, Jennison AV. Characterisation of invasive clinical Haemophilus influenzae isolates in Queensland, Australia using wholegenome sequencing. Epidemiol Infect. 2017;145(8):1727-36.

14. Pinto M, Gonzalez-Diaz A, Machado MP, Duarte S, Vieira L, Carrico JA, et al. Insights into the population structure and pan-genome of Haemophilus influenzae. Infect Genet Evol. 2019;67:126-35.

15. Kroll JS, Zamze S, Loynds B, Moxon ER. Common organization of chromosomal loci for production of different capsular polysaccharides in Haemophilus influenzae. J Bacteriol. 1989;171(6):3343-7.

16. Kroll JS, Loynds B, Brophy LN, Moxon ER. The bex locus in encapsulated Haemophilus influenzae: a chromosomal region involved in capsule polysaccharide export. Mol Microbiol. 1990:4(11):1853-62.

17. Kroll JS, Hopkins I, Moxon ER. Capsule loss in H. influenzae type b occurs by recombination-mediated disruption of a gene essential for polysaccharide export. Cell. 1988;53(3):347-56.

18. Sukupolvi-Petty S, Grass S, St Geme JW 3rd. The Haemophilus influenzae type $b$ hcsA and hcsB gene products facilitate transport of capsular polysaccharide across the outer membrane and are essential for virulence. J Bacteriol. 2006;188(11):3870-7.

19. Follens $A$, Veiga-da-Cunha M, Merckx R, van Schaftingen E, van Eldere J. acs1 of Haemophilus influenzae type a capsulation locus region II encodes a bifunctional ribulose 5-phosphate reductase- CDP-ribitol pyrophosphorylase. J Bacteriol. 1999;181(7):2001-7.

20. Van Eldere J, Brophy L, Loynds B, Celis P, Hancock I, Carman S, et al. Region II of the Haemophilus influenzae type be capsulation locus is involved in serotype-specific polysaccharide synthesis. Mol Microbiol. 1995;15(1):107-18.

21. Lam $T$, Claus H, Frosch M, Vogel U. Sequence analysis of serotype-specific synthesis regions II of Haemophilus influenzae serotypes $\mathrm{c}$ and d: evidence for common ancestry of capsule synthesis in Pasteurellaceae and Neisseria meningitidis. Res Microbiol. 2011;162(5):483-7.

22. Giufre M, Cardines R, Mastrantonio P, Cerquetti M. Genetic characterization of the capsulation locus of Haemophilus influenzae serotype e. J Clin Microbiol. 2010;48(4):1404-7.

23. Lam TT, Elias J, Frosch M, Vogel U, Claus H. New diagnostic PCR for Haemophilus influenzae serotype e based on the cap locus of strain ATCC 8142. Int J Med Microbiol. 2011:301(2):176-9.

24. Satola SW, Schirmer PL, Farley MM. Genetic analysis of the capsule locus of Haemophilus influenzae serotype f. Infect Immun. 2003;71(12):7202-7.

25. Wroblewski D, Halse TA, Hayes J, Kohlerschmidt D, Musser KA. Utilization of a real-time $P C R$ approach for Haemophilus influenzae serotype determination as an alternative to the slide agglutination test. Mol Cell Probes. 2013;27(2):86-9.

26. Falla TJ, Crook DW, Brophy LN, Maskell D, Kroll JS, Moxon ER. PCR for capsular typing of Haemophilus influenzae. J Clin Microbiol. 1994:32(10):2382-6.

27. Gonin P, Lorange M, Delage G. Performance of a multiplex PCR for the determination of Haemophilus influenzae capsular types in the clinical microbiology laboratory. Diagn Microbiol Infect Dis. 2000;37(1):1-4.

28. LaClaire LL, Tondella ML, Beall DS, Noble CA, Raghunathan PL, Rosenstein $N E$, et al. Identification of Haemophilus influenzae serotypes by standard slide agglutination serotyping and PCR-based capsule typing. J Clin Microbiol. 2003;41(1):393-6.

29. Lam $\Pi$, Claus H, Frosch M, Vogel U. Analysis of non-typeable Haemophilus influenzae in invasive disease reveals lack of the capsule locus. Clin Microbiol Infect. 2016;22(1):63 e7- e8.

30. Maaroufi Y, De Bruyne JM, Heymans C, Crokaert F. Real-time PCR for determining capsular serotypes of Haemophilus influenzae. J Clin Microbiol. 2007:45(7):2305-8.

31. Erwin AL, Sandstedt SA, Bonthuis PJ, Geelhood JL, Nelson KL, Unrath WC, et al. Analysis of genetic relatedness of Haemophilus influenzae isolates by multilocus sequence typing. J Bacteriol. 2008;190(4):1473-83.
32. Theodore MJ, Anderson RD, Wang X, Katz LS, Vuong JT, Bell ME, et al. Evaluation of new biomarker genes for differentiating Haemophilus influenzae from Haemophilus haemolyticus. J Clin Microbiol. 2012;50(4): $1422-4$.

33. Watts SC, Holt KE. hicap: In Silico Serotyping of the Haemophilus influenzae Capsule Locus. J Clin Microbiol. 2019;57(6). https://doi.org/10.1128/JCM. 00190-19. Print 2019 June.

34. Vuong J, Collard JM, Whaley MJ, Bassira I, Seidou I, Diarra S, et al. Development of real-time PCR methods for the detection of bacterial meningitis pathogens without DNA extraction. PLoS One. 2016;11(2): e0147765.

35. Wang X, Mair R, Hatcher C, Theodore MJ, Edmond K, Wu HM, et al. Detection of bacterial pathogens in Mongolia meningitis surveillance with a new real-time PCR assay to detect Haemophilus influenzae. Int J Med Microbiol. 2011:301(4):303-9.

36. Martin M. Cutadapt removes adapter sequences from high-throughput sequencing reads. EMBnet. 2011;17(1):3.

37. Bankevich A, Nurk S, Antipov D, Gurevich AA, Dvorkin M, Kulikov AS, et al. SPAdes: a new genome assembly algorithm and its applications to singlecell sequencing. J Comput Biol. 2012;19(5):455-77.

38. Kretz CB, Retchless AC, Sidikou F, Issaka B, Ousmane S, Schwartz S, et al. Whole-genome characterization of epidemic Neisseria meningitidis Serogroup C and resurgence of Serogroup W, Niger, 2015. Emerg Infect Dis. 2016;22(10):1762-8.

39. Gardner SN, Slezak T, Hall BG. KSNP3.0: SNP detection and phylogenetic analysis of genomes without genome alignment or reference genome. Bioinformatics. 2015;31(17):2877-8.

40. Letunic I, Bork P. Interactive tree of life (iTOL) v3: an online tool for the display and annotation of phylogenetic and other trees. Nucleic Acids Res. 2016;44(W1):W242-5.

41. Jolley KA, Maiden MC. BIGSdb: scalable analysis of bacterial genome variation at the population level. BMC Bioinformatics. 2010;11:595.

42. Huson DH, Bryant D. Application of phylogenetic networks in evolutionary studies. Mol Biol Evol. 2006;23(2):254-67.

43. Edgar RC. MUSCLE: multiple sequence alignment with high accuracy and high throughput. Nucleic Acids Res. 2004;32(5):1792-7.

44. Altschul SF, Madden TL, Schaffer AA, Zhang J, Zhang Z, Miller W, et al. Gapped BLAST and PSI-BLAST: a new generation of protein database search programs. Nucleic Acids Res. 1997;25(17):3389-402

45. Jones P, Binns D, Chang HY, Fraser M, Li W, McAnulla C, et al. InterProScan 5: genome-scale protein function classification. Bioinformatics. 2014;30(9): $1236-40$.

46. Cock PJ, Antao T, Chang JT, Chapman BA, Cox CJ, Dalke A, et al. Biopython: freely available Python tools for computational molecular biology and bioinformatics. Bioinformatics. 2009;25(11):1422-3.

\section{Publisher's Note}

Springer Nature remains neutral with regard to jurisdictional claims in published maps and institutional affiliations.

Ready to submit your research? Choose BMC and benefit from

- fast, convenient online submission

- thorough peer review by experienced researchers in your field

- rapid publication on acceptance

- support for research data, including large and complex data types

- gold Open Access which fosters wider collaboration and increased citations

- maximum visibility for your research: over $100 \mathrm{M}$ website views per year

At BMC, research is always in progress.

Learn more biomedcentral.com/submission 